\title{
Scanning the steel 08G2B using superminiature eddy current probe
}

\author{
Sergey Dmitriev ${ }^{1, *}$, Alexey Ishkov $^{2}$, Vladimir Malikov ${ }^{1}$, Alexander Katasonov $^{1}$, Alexey \\ Grigorev $^{1}$, Mihail Ryasnoy ${ }^{1}$ and Anatoly Sagalakov ${ }^{1}$ \\ ${ }^{1}$ Altai State University,61 Lenina Av., Barnaul, 656049, Russia \\ ${ }^{2}$ Altai State Agricultural University, 98 Krasnoarmeyskiy Av., Barnaul, 656049, Russia
}

\begin{abstract}
The article describes a hardware and software complex designed for defectoscopy and study of material properties. Sections of high-strength steel 08G2B pipes were scanned in the course of the research. The purpose of this study is to determine dependency of eddy current sensor signal and mechanical and operational properties of the base metal (K65 steel grade) and demonstrate the ability of developed eddy current probe to detect continuity defects and corrosion in this type of metal. The steel is ultra-low-carbon, which makes it possible to study their properties using the eddy current method. The eddy current method is a non-contact one being used both for cracks identification and determination of their characteristics. A special superminiature eddy current probe was developed, which includes three coils - stabilizing, measuring and exciting. The coils were placed on a pyramid-shaped core made of alloy 81NMA. The eddy-current transducer runs under the control of the developed hardware-software complex with a system for suppression of noise and amplification of signal received from the eddycurrent transducer. Signal processing makes it possible to separate the effects of closely spaced cracks on the eddy-current transducer signal and evaluate the contribution of each crack separately. As a parameter containing information about the presence of defects in the object of control, the voltage arrived at the measuring coil of the eddy current probe signal was used. The work studied sections of pipes with model continuity defects - cracks and holes. A research that allows to simulate the presence of corrosion in the metal was also conducted. The extreme values of the eddy current probe signal over defects of different types are obtained at various velocity, that allows to identify the type of defect, while changing the frequency of the signal on the probe allows to determine the depth of the defect.
\end{abstract}

\section{Introduction}

Weathering steels, atmospheric corrosion resistant steels, which are low alloy high strength steels containing a small amount of alloying elements, have a good corrosion resistance performance in atmosphere, lower cost compare with stainless steels. Weathering steels

*Corresponding author: osys@me.com 
have widely used in bridges, buildings, vehicles, container, transmission tower, and other industries on account of their excellent atmospheric corrosion resistance.

One of the current fields of use of high-strength steel in construction is pipelines for various applications (main pipelines, process pipelines, etc.). Problems of ensuring the reliability and fail-safety of steel pipelines due to their susceptibility to normal aging is very serious.

Generally, failures of pipelines are caused by corrosion and mechanical fatigue of metal.

The facility structures such as oil/gas tanks and pipes are often made of steel materials. With elapse of usage years, their state deteriorates due to some defects of corrosion, crack, wall thinning, etc. Finally, it may worsen to reach an accident. Therefore, their preventive diagnosis is an important issue for facility safety.

Modern pipeline steels used for the transportation of crude oil and natural gas over large distances under high pressure, need to satisfy the requirements specified by the American Petroleum Institute (API). These grades are processed by thermo-mechanical controlled rolling (TMCR) and accelerated cooling route with the prime aspiration to achieve the best possible combination of strength and toughness [1].

Besides high strength, high fracture toughness and impact toughness are essential to prevent premature ductile and brittle failure, while formability is required for pipe-bowing.

Good weldability, resistance to hydrogen induced blister cracking in sour service environment, resistance to stress corrosion cracking resistance for underground service, especially in $\mathrm{H} 2 \mathrm{~S}$ environment, and fatigue resistance are the additional requirements for pipeline steels.

During long-term services, corrosion might occur on the outer side of the pipe as corrosion under insulation (CUI), or on the inner side of the pipe as flow accelerated corrosion (FAC). Wall thinning of pipelines can develop, which may finally results in a catastrophic failure. Therefore, local wall thinning is a point of concern in almost all steel structures, such as pipelines, particularly pipelines which are covered with a thermal insulator made of materials having low thermal conductivity (fiberglass or mineral wool). Hence, the NDT methods which are capable of detecting wall thinning and defects without removing the insulation are necessary.

One of typical methods to detect metal state is eddy current testing, that is widely used for defect inspection of metal surface such as in aircraft components and heat exchanger tube testing, etc. Advantage of the eddy current testing is that it is noncontact, high speedy, automatic implementable, excellent to surface detection, tolerant to high temperature and pressure. On the other hand, its disadvantage is that it is difficult to inspect internal state of thick material and is not applicable to ferromagnetic metals [1], [2].

Several researchers have examined the EC techniques for metallurgical characterization of different alloys [3-5]. For example, Pearlite volume fraction measuring of plain carbon steel showed that there is a suitable relation between EC output (impedance) and pearlite percent. In addition, applicability of EC technique to characterize austenitic variation during cold rolling of stainless steels was reviewed. It was observed that EC indexes are affected due to the variation of volume fraction of austenite. Therefore, it was confirmed that the EC measurement technique can be a useful tool for the recognition of austenite variation during the cold rolling of stainless steels [3].

Using EC techniques for measuring the roughness parameters of ferrous and nonferrous metals was examined. The results confirmed the applicability of the EC technique for measuring the roughness parameters both under static and dynamic conditions. A research on the dual phase steel (DPS) revealed that a good correlation exists between EC outputs with microstructural and mechanical changes in the DPS specimens $(\mathrm{R} 2>0.85)$. It showed the ability of the EC technique for prediction of material properties in the DPS [4]. The variation of ferrite grain size on impedance (EC output) was also studied. The results reveal 
that this technique has a high potential to be used as a reliable NDT tool to detect and to monitor the microstructural changes during the manufacturing of DPS [5].

The pulsed eddy current technique (ECT) uses repetitive pulses having a short duration in time instead of a sinusoidal wave with a single frequency. A pulsed excitation generates numerous frequencies simultaneously in the work piece [6]. The ECT technique offers an alternative to these conventional techniques due to its potential advantages, such as less susceptible to interference, less power consumption because of using short pulses which are more desirable specification in the development of portable instruments. The conventional ECT which operateswith a single frequency sinusoidal excitation has gained wide acceptance in the field of NDT [7]; yet, this technique suffers from a limitation, i.e., penetration depth or skin depth. The skin depth equation is given by, where $\mu$ is the permeability, $\sigma$ is the conductivity and $f$ is the frequency of excitation, and the penetration depth $\delta$ depends on excitation frequency $\mathrm{f}$ [8]. Because the Fourier transform of a pulse contains multiple frequency components $[9,10]$, a rectangular pulse can provide the depth profile of a material under test [11]. The usage of short current pulse excitation reduces the power consumption, which is the most desired specification in the development of portable instruments. Due to the potential advantages of the ECT, prevalent investigations on this technique have been conducted, such as detection of wall thinning and corrosion in aircraft multilayer structures $[12,13]$. On the other hand, many parts of the pipelines in nuclear power plants are welded with a dissimilar metal. The ECT techniques are expected to detect the defects in the dissimilar weld part. Analytical expression for transient induction voltage of receiving coil created by the pulsed eddy current induced by a transmitting coil over a conducting plate was presented elsewhere [14]. ECT testing has been applied to pipeline inspection by some leading inspection companies [15].

The purpose of this study is to determine dependency of eddy current sensor signal and mechanical and operational properties of the base metal (K65 steel grade) and demonstrate the ability of developed eddy current probe to detect continuity defects and corrosion in this type of metal.

\section{Materials and methods}

Steel pipes with model defects made of high-strength steel 08G2B were used as an object of the study. Cracks were simulated as model defects, as well as pointlike corrosion damages made by drilling metal to different depths.

In accordance with previous studies [16-18], a design of an eddy current probe was proposed, which consists of a pyramid-shaped magnetic core made of permalloy 81NMA. The magnetic core was wound with an exciting, a measuring and a stabilizing coils. Turns of the coils were impregnated with epoxy compounds and formed a monolithic structure.

At the manufacturing stage, the initial adjustment is performed, according to which the amplitude and phase data are written into the computing unit, the coefficients of the inverse transformation functions are determined, which correspond to the thicknesses of the control samples at fixed values of the gap between the surface of the pipe and the eddy current probe (ECP). Tuning is performed in a laboratory environment. Before you start working with the device, a working tuning is performed, within which the ECP is balanced in the absence of the test object and the amplitude of the first frequency is recorded at zero clearance between the ECP and the surface of the test object, which is used in the calculation function of the gap; the deviation of the instrument readings from the actual value is checked and automatic correction is performed. The ECP waybill class today is represented by a large number of diverse designs that solve a wide range of non-destructive testing tasks. 
The frequency of the excitation current was taken equal to 500 and $2000 \mathrm{~Hz}$. The frequencies were selected as follows: for the lower frequency, the depth of penetration of the magnetic field into the test object should be greater than the values of the measured wall thickness, for the top, the depth of penetration into the metal should be less than the value of the wall thickness, while sufficient to ensure sensitivity to changes in the electrical conductivity of the material and the gap between ECP and control surface. The physical meaning of the penetration depth is as follows: in a layer of depth $\delta$, the entire current excited in the corresponding section of the metal would be concentrated if its density in this layer was equal to the maximum density $\mathrm{J}_{\max }$ of the eddy current generated on the surface. The penetration depth $\delta$ of eddy currents for a plane wave is equal to the depth at which the eddy current density decreases e times compared to the density on the metal surface from the side of the source of alternating magnetic field.

\section{Result and discussion}

A steel pipe with milled cracks was used as a sample No. 1. The thickness of the pipe was 8 $\mathrm{mm}$. The cracks were located at a depth of 1,3 and $5 \mathrm{~mm}$ (Fig. 1) and had an opening width of $2 \mathrm{~mm}$. The current frequency on the exciting coil was $500 \mathrm{~Hz}$.

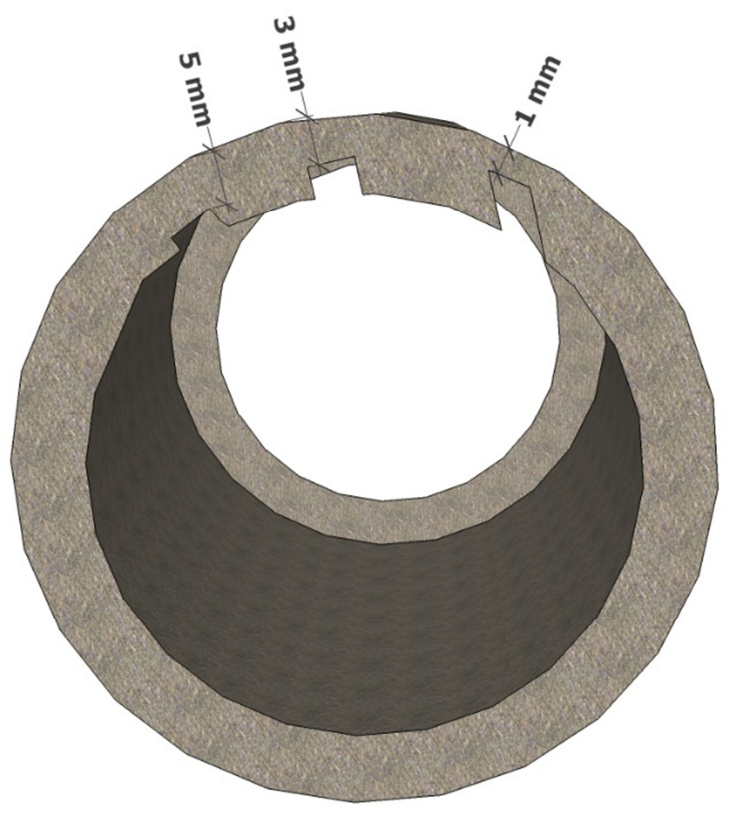

Fig. 1. Steel pipe with milled cracks

The dependence of signal amplitude on the probe location above the inspected object shows the location of defects via amplitude drop (Fig.2.). 1 - corresponds to crack located at 1 $\mathrm{mm}$ depth, 2 - a crack at $3 \mathrm{~mm}$ depth, 3 - a crack at $5 \mathrm{~mm}$ depth. 


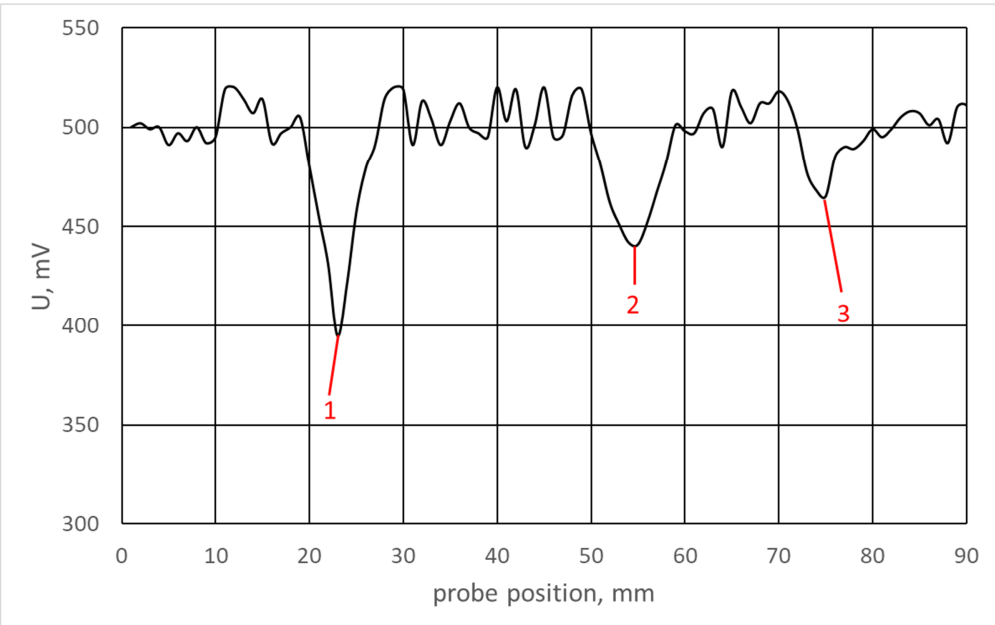

Fig. 2. Results of a pipe inspecting at frequency of $500 \mathrm{~Hz}$ (type defects - crack)

The graph shows a drop in signal amplitude that begins long before the scanning sensor approaches the crack. The middle of the crack in metal corresponds to the lowest value of the signal amplitude, which allows to determine its location. In this case, the dependence of the signal amplitude on the position of the probe relative to the first crack can be described by the following function:

$$
U=-0.2273 x^{4}+21.03 x^{3}-720.34 x^{2}+10820 x-59664
$$

By changing the operating frequency of the probe, it is possible to determine the depth of the crack. Fig. 3 shows a graph of the signal amplitude change at frequencies of $2000 \mathrm{~Hz}$ (Line 1) and $5000 \mathrm{~Hz}$ (Line 2). Only two defects can be determined by changing the amplitude of line 1, and one defect can be determined by changing the amplitude of line 2 . Using the known formula for the depth of penetration of eddy currents, it is possible to use the results of scanning at different frequencies to determine the depth of cracks.

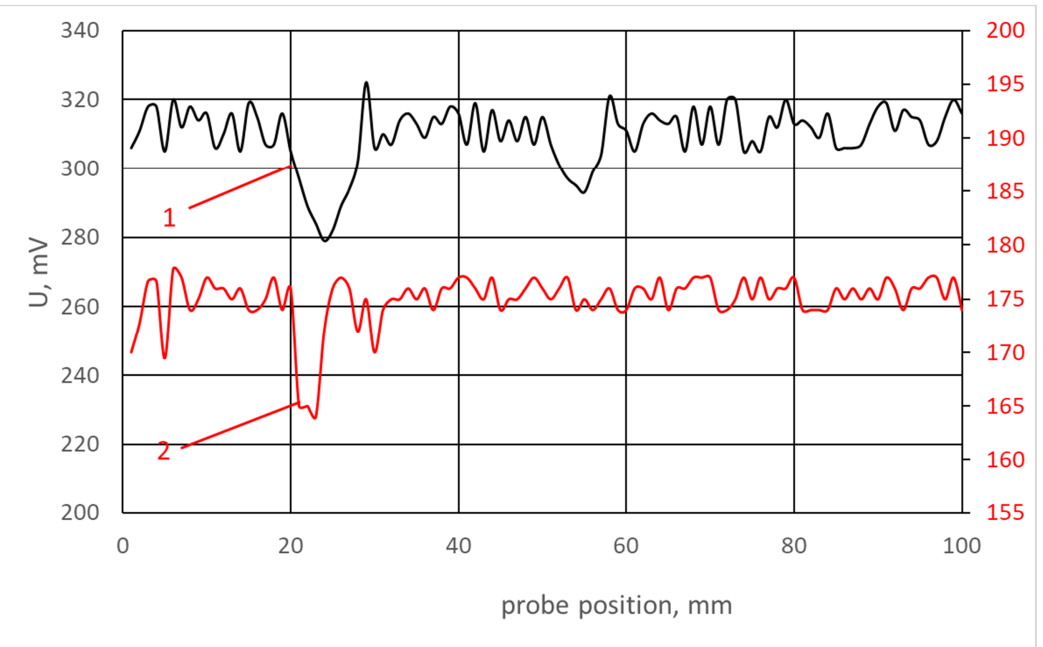

Fig. 3. Results of a pipe inspecting at frequency of $2,000 \mathrm{~Hz}$ (line 1) and 5,000 $\mathrm{Hz}$ (line 2)

Sample No. 2 was a steel pipe with three drilled holes at a depth of $3 \mathrm{~mm}$. 
A steel pipe with three drilled holes with a diameter of 1, 3 and $5 \mathrm{~mm}$ was used as a sample No. 1. The dependence of the output voltage of the probe on the position of the probe shows the location of the hole by the drop in the voltage amplitude (Fig. 4). Compared to fig. 3 it is possible to note a steeper drop in the probe voltage when approaching the defect. The dependence of the probe signal voltage on its position when approaching the first hole can be described by the following function:

$$
\mathrm{U}=69 \mathrm{x}^{2}-4277 \mathrm{x}+66718
$$

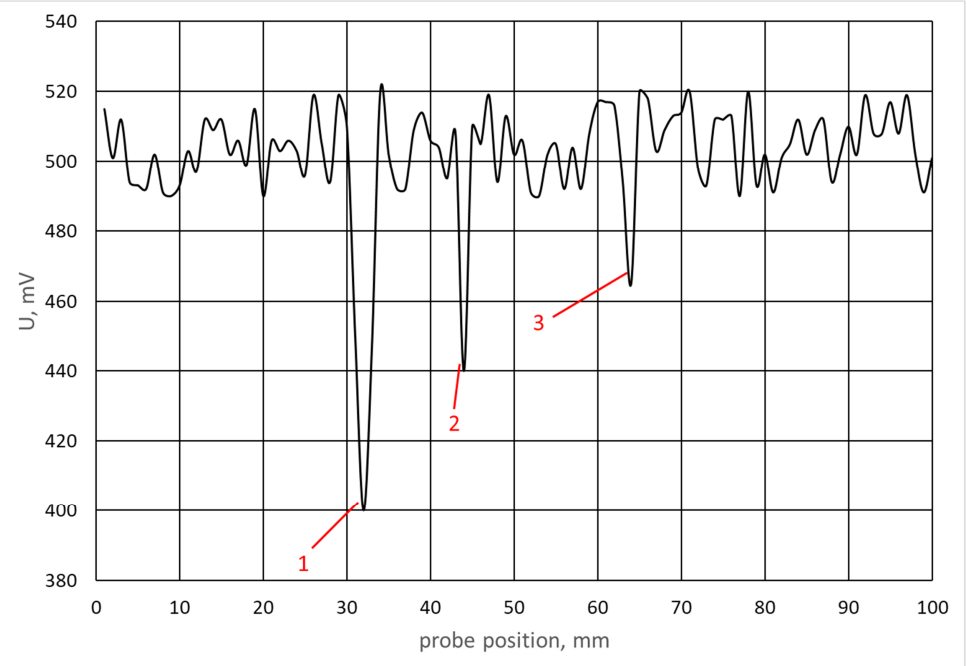

Fig. 4. Results of a pipe inspecting at frequency of $500 \mathrm{~Hz}$ (type defects - hole)

\section{Conclusion}

The test results of the developed hardware and software complex demonstrated its significant effectiveness in searching for such defects of steel pipes as stress corrosion cracks and holes in metal. The extreme values of the eddy current probe signal over defects of different types are obtained at various velocity, that allows to identify the type of defect, while changing the frequency of the signal on the probe allows to determine the depth of the defect. At the same time, the developed eddy current probe is able to study other metallic ferromagnetic and non-ferromagnetic materials.

\section{References}

1. K.H. Lee, I.H. Park. 2012 Sixth International Conference on Electromagnetic Field Problems and Applications, 1, 1-5 (2012) DOI: 10.1109/ICEF.2012.6310321

2. J.C. Moulder, B.A J. Nondestruc. Eval., 18, 3-7 (1999) DOI: 10.1023/A:1021898520626

3. S.H. Khan, F. Ali, A. Nusair Khan, M.A. Iqbal. J. Mat. Pro. Tech., 200, 316-318 (2008) DOI: 10.1016/j.jmatprotec.2007.09.003

4. S.H. Khan, F. Ali, A. Nusair Khan, M.A. Iqbal. J. Computational Materials Science. 43, 623-628 (2008) DOI: 10.1016/j.commatsci.2008.01.034

5. Y. Bilik, D. Bilik, A. Moshkovich, V. Perfiyev, L. Rapoport. Materials Evaluation, 69(6), 794-802 (2011). 
6. I.Z. Abidin, C. Mandache. NDT\&E International, 42, 599-605 (2009) DOI: 10.1016/j.ndteint.2009.04.001

7. N. Nair, V. Melapudi, L. Udpa, X. Liu, A GMR-Based Eddy Current System for NDE of Aircraft Structures. IEEE Trans. Magn., 42(10), 3312-3314 (2006) DOI: 10.1109/TMAG.2006.879820

8. R. Grimberg, L. Udpa, A. Savin, R. Steigmann, V. Palihovic. NDT\&E Int., 39, 264 (2006) DOI: 10.1016/j.ndteint.2005.08.004

9. A. Sophian, G. Y. Tian, D. Taylor. NDT \& E int, 36, 37-45 (2003) DOI: 10.1016/S0963-8695(02)00069-5

10. B Hu, $\mathrm{R} \mathrm{Yu}, \mathrm{H}$ Zou. NDT\&E International, 47, 66-69 (2012) DOI: 10.1016/j.ndteint.2011.12.007

11. T Santos, L Rosado. Innovative concept and application of ec probe for inspection of friction stir welds. The 12th International Conference of the Slovenian Society for NonDestructive Testing »Application of Contemporary Non-Destructive Testing in Engineering», 1, 329-338 (2013)

12. M. A. Robers and R. S. Scottini. Pulsed eddy current in corrosion detection. Proc. of 8th ECNDT conf, ndt.net, $\quad \mathbf{7 ( 1 0 ) ,} \quad 329 \quad$ (2002) https://www.ndt.net/article/ecndt02/251/251.htm

13. R. A. Smith and G. R. Hugo. Insight, 14(1), 14-25 (2001) https://www.ndt.net/publicat/bibliog/insi2001.htm

14. V. O. de Haan and P. A. de Jong. Analytical expressions for transient induction voltage in a receiving coil due to a coaxial transmitting coil over a conducting plate. IEEE, Trans. Magn., 40, 371-378 (2004) DOI: 10.1109/TMAG.2004.824100

15. D. G. Park, C. S. Angani. Journal of Magnetics, 18, $342-347$ (2013) DOI: 10.4283/JMAG.2013.18.3.342

16. S F Dmitriev, V N Malikov, A M Sagalakov, M A Davydchenko. Subminiature eddycurrent transducers for conductive materials research. Proceedings of 2015 International Conference on Mechanical Engineering, Automation and Control Systems, MEACS 2015, 1, 1-5 (2016) DOI: 10.1109/MEACS.2015.7414951

17. V N Malikov, S F Dmitriev. Russ. Eng. Res., 8(1), 626-629 (2016) DOI: 10.3103/S1068798X16080074

18. S F Dmitriev, V N Malikov, A M Sagalakov. Welding International, 31(8), 608-611 (2017) DOI: 10.1080/09507116.2017.1295563 